\title{
Intra-module Inference
}

\author{
Shuvendu K. Lahiri ${ }^{1}$, Shaz Qadeer ${ }^{1}$, Juan P. Galeotti ${ }^{2}$, Jan W. Voung ${ }^{3}$, \\ and Thomas Wies ${ }^{4}$ \\ ${ }^{1}$ Microsoft Research \\ ${ }^{2}$ University of Buenos Aires \\ ${ }^{3}$ University of California, San Diego \\ ${ }^{4}$ École Polytechnique Fédérale de Lausanne
}

\begin{abstract}
Contract-based property checkers hold the potential for precise, scalable, and incremental reasoning. However, it is difficult to apply such checkers to large program modules because they require programmers to provide detailed contracts, including an interface specification, module invariants, and internal specifications. We argue that given a suitably rich assertion language, modest effort suffices to document the interface specification and the module invariants. However, the burden of providing internal specifications is still significant and remains a deterrent to the use of contract-based checkers. Therefore, we consider the problem of intra-module inference, which aims to infer annotations for internal procedures and loops, given the interface specification and the module invariants. We provide simple and scalable techniques to search for a broad class of desired internal annotations, comprising quantifiers and Boolean connectives, guided by the module specification. We have validated our ideas by building a prototype verifier and using it to verify several properties on Windows device drivers with zero false alarms and small annotation overhead. These drivers are complex; they contain thousands of lines and use dynamic data structures such as linked lists and arrays. Our technique significantly improves the soundness, precision, and coverage of verification of these programs compared to earlier techniques.
\end{abstract}

\section{Introduction}

Program verification is an undecidable problem, which makes it impossible to build automated and precise program verifiers. In the last few decades, research in static analysis and program verification has attempted to improve the precision and scalability of program verification tools without requiring significant user input. Most existing tools for verifying properties of software fall under two extremes: push-button tools based on model checking [7] and abstract interpretation 9 that have little room for user guidance, or contract-based verifiers such as ESC/Java [14] and Spec\# [5] that require the user to specify all the contracts.

Contract-based property checkers hold the potential for precise, scalable, and incremental reasoning. However, it is difficult to apply such checkers to large program modules because they require programmers to provide detailed contracts.

A. Bouajjani and O. Maler (Eds.): CAV 2009, LNCS 5643, pp. 493 508, 2009.

(C) Springer-Verlag Berlin Heidelberg 2009 
These contracts include an interface specification (preconditions and postconditions for public procedures), module invariants, and internal specifications (loop invariants, preconditions, and postconditions for internal procedures). Manually providing such contracts is infeasible for large modules that typically contain thousands of lines and hundreds of procedures. Operating systems modules such as device drivers, file systems, and memory managers are typically large and consequently remain outside the scope of existing verification techniques.

We argue that given a suitably rich assertion language, modest effort suffices to document the interface specification and the module invariants. This is fortunate because these specifications are the most useful for documentation and program understanding. While the interface specification documents the client's view, the module invariants provide the central argument for establishing the interface specification and other desirable properties of the module. However, the burden of providing internal specifications is still significant and remains a deterrent to the use of contract-based checkers. Therefore, we solve the problem of intra-module inference. Given a module with an interface specification, module invariants, and a property to be proved, we infer annotations on loops and internal procedures guided by the provided specifications.

In this work, we demonstrate how to synthesize a broad class of internal annotations containing quantifiers and Boolean structure, in a scalable fashion, guided by the module invariants. Our inference method generates a set of candidate annotations using an idea of exception sets, and then searches for annotations within the candidate pool using the scalable Houdini algorithm [13. We formalize our ideas in terms of a general and extensible annotation language comprising type-state assertions. The type-state of a pointer can be static or can depend on runtime attributes such as the runtime type of a pointer, values of object fields, and membership (or non-membership) in heap-allocated data structures 22 .

We have validated our ideas by building a prototype verifier and using it to verify several properties on Windows device drivers with zero false alarms and small annotation overhead. These drivers are complex; they contain thousands of lines and use dynamic data structures such as linked lists and arrays. We show that proving even simple type-state properties may require tracking typestates related to linked lists and non-trivial aliasing constraints in the module invariants. We then demonstrate how our inference technique is able to infer almost all internal annotations. Our technique significantly improves the soundness, precision and coverage of verification of these programs compared to earlier techniques applied on these programs (see Section 6 for related work).

Our experience leads us to the following conclusions:

1. Having the programmer specify the module invariants and the tool infer the internal annotations is a useful tradeoff in the quest for automated program verifiers that can check general properties with high precision. Given only the property to be proved, inferring the module invariants automatically with reasonable cost seems unlikely because the required invariant may depend on sophisticated type-state abstractions absent from the property. Inference of 
internal annotations guided by the structure of the module invariants seems more amenable to cost-effective automation.

2. Searching for internal annotations guided by user-specified module invariants discovers annotations that are understandable by a programmer, unlike intermediate assertions of static analysis tools that can only be understood by machines. This attribute is important if a tool attempts to aid program documentation and incremental checking, in addition to finding bugs.

\section{Motivating Example}

In this section, we show the module invariants and internal annotations required to verify properties of a real-life Windows device driver kbdclass. We focus on checking the absence of the following double-free property: a pointer of type DEVICE_OBJECT that is deleted with a call to IoDeleteDevice was allocated via a prior call to IoCreateDevice, and an object is not deleted twice. The purpose of this section is to show that the module invariants required to check this property can be expressed succinctly over a set of suitable type-states with a relatively low annotation burden. On the other hand, it is non-trivial to arrive at the relevant invariants mechanically starting from the property of interest, as they contain type-state abstractions, quantifiers and Boolean structure.

\subsection{Module Invariants}

Module invariants are a set of assertions that are preserved by the public procedures of a module and are strong enough to prove the property of interest. Figure 2 shows the module invariants required to prove the double-free property on kbdclass. Each of these invariants either specifies a property on all pointers satisfying a dynamic type-state, or the type-state of a global variable. In this section, we will explain these invariants with respect to the kbdclass module.

Allocation type-states and aliasing invariants. We use the mutable sets MyDevObj and MyDevExtn to model the allocation (in IoCreateDevice) and deletion (in IoDeleteDevice) of DEVICE_OBJECT pointers in kbdclass. The structure of a device object and a device extension are described below (ignore the field of type PLISTENTRY for now):

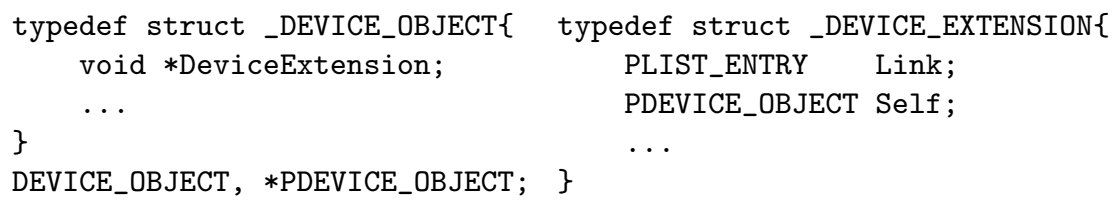

DEVICE_EXTENSION, *PDEVICE_EXTENSION;

Intuitively, each call to IoCreateDevice adds a pointer $u$ to MyDevObj and a pointer $v$ to MyDevExtn, setting $u->$ DeviceExtension to $v$ at the same time. Conversely, when a pointer $u$ is passed to IoDeleteDevice, it removes $u$ from MyDevObj and $u->$ DeviceExtension from MyDevExtn. The aliasing constraints 


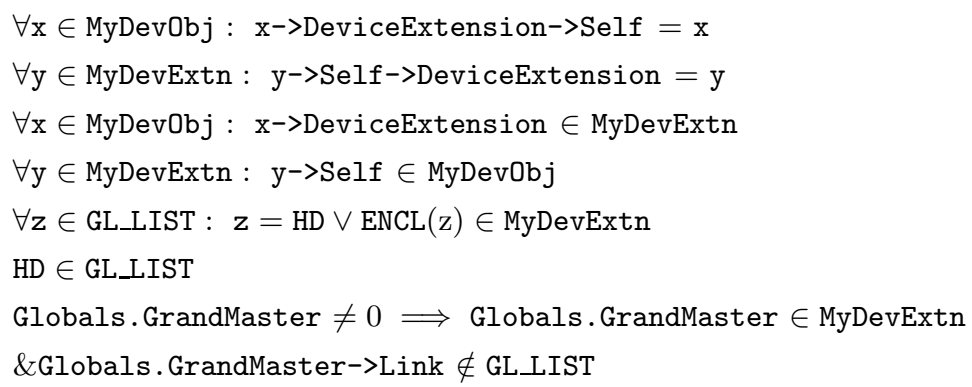

Fig. 2. Module invariants for checking double-free property on kbdclass. ENCL ( $x$ ) is a macro for CONTAINING_RECORD (x, DEVICE_EXTENSION, Link).

The GLOBALS structure contains a pointer to a device extension in GrandMaster, and the head of a list of device extensions in LegacyDeviceList. The LIST_ENTRY structure contains forward and backward links for a linked list; fields of this type represent the head (LegacyDeviceList in GLOBALS) as well as the links (Link in DEVICE_EXTENSION) in a list (refer to Figure 1).

Now consider the public procedure KeyboardClassUnload which deletes all the device objects present in the driver, before unloading the driver.

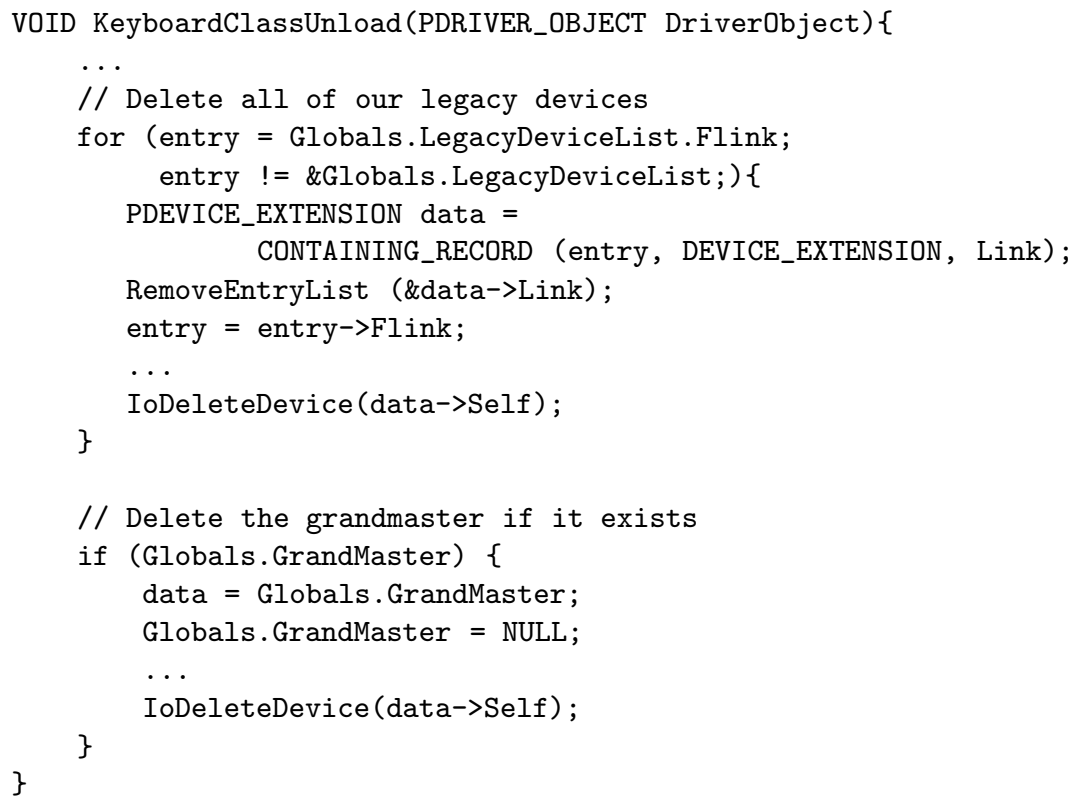

The loop in the procedure iterates over the list Globals.LegacyDeviceList using the iterator variable entry. The macro CONTAINING_RECORD is used to obtain the enclosing structure given the address of an internal field, and is defined as: 
\#define CONTAINING_RECORD $(x, T, f)((T *)(($ int $)(x)-($ int $)(\&((T$ *)0) $->f$ )))

For each iteration, data points to the enclosing DEVICE_EXTENSION structure of entry. This entry is removed from the list by RemoveEntryList and freed by the call to IoDeleteDevice on data->Self. Finally, the device object in Globals.GrandMaster is freed, after the loop terminates.

We argue about the safety of these operations in the remainder of this section. First, we define two macros that are used in the definition of the module invariants in Figure 2, to refer to the linked list in Globals . LegacyDeviceList:

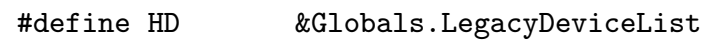

Here HD denotes the dummy head of the linked list and GL_LIST denotes the pointers in the linked list. The set constructor Btwn $(\mathrm{f}, x, y)$ [20] denotes the set of pointers $\{x, x->\mathbf{f}, x->\mathbf{f}->\mathbf{f}, \ldots, y\}$ when $x$ reaches y using $\mathrm{f}$, or \{\} otherwise.

1. We need to specify that for each entry $z$ of the list apart from the dummy head HD, the pointer CONTAINING_RECORD ( $z, \operatorname{DEVICE\_ EXTENSION,~Link)~is~}$ a MyDevExtn pointer. This is specified in invariant 5. Invariant 6 denotes that the set is non-empty. Notice that invariant 5 corresponds to a typestate invariant, where the type-state is determined by membership in a data structure.

2. For any MyDevExtn pointer $x, x->$ Self should be a MyDevObj pointer, to enable the call to IoDeleteDevice to succeed (invariant 4).

3. Furthermore, we need to ensure that IoDeleteDevice is not invoked on the same device object pointer in two different iterations of the loop. This can happen if $x->\operatorname{Self}=y->\operatorname{Self}$, where $x$ and $y$ correspond to the values of data in two different loop iterations. This can be prevented by requiring that any MyDevExtn pointer $x$ satisfies $x->$ Self->DeviceExtension $=x$ (invariant 2).

4. Finally, invariant 7 ensures that Globals.GrandMaster is a valid device extension and invariant 8 ensures that Globals. GrandMaster is not present in the GL_LIST, and therefore Globals.GrandMaster->Self was not freed in the loop.

\subsection{Internal Annotations}

Let us now look at some of the annotations required on the internal procedures to verify the absence of the double-free property modularly. In the ideal case, the module invariants would act as the necessary preconditions, postconditions and loop invariants for all the procedures. However, these invariants can be temporarily broken due to allocation of new objects satisfying a given type-state or mutation of fields in objects. For instance, many invariants on the global state (invariants 5. 7. 8) do not hold on internal procedures and loops called within the initialization procedure of the module. For kbdclass, most of the internal 
annotations in addition to the module invariants can be categorized into one of the following:

Module invariant exceptions. In the procedure KeyboardClassUnload below, a device object $*$ ClassDeviceObject is initialized after being created by IoCreateDevice.

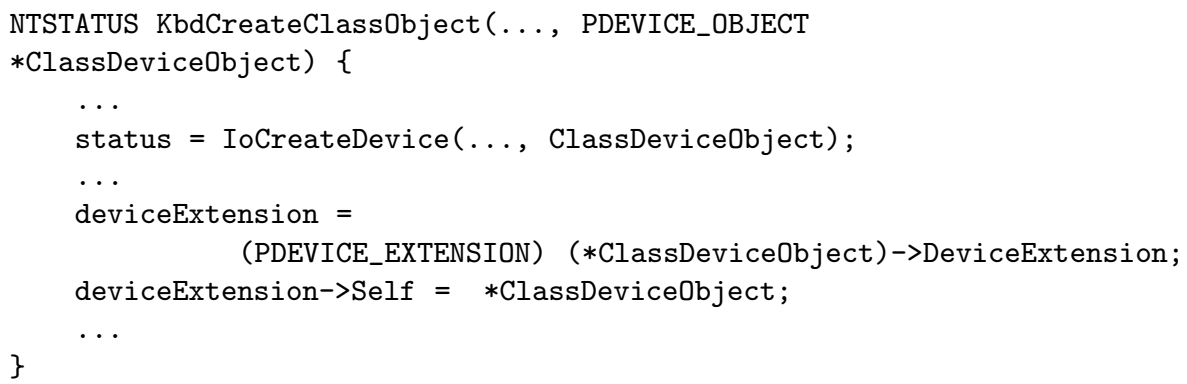

Any procedure call between the call to IoCreateDevice and the setting of Self would violate the invariant 1. In this case, the type-state invariant 1 holds for all pointers with the exception of $*$ ClassDeviceObject.

Type-states of pointers and their fields. We have to propagate the typestates of pointers available from preconditions of public procedures to internal procedures to utilize the type-state invariants. In another case, we need the loop invariant entry $\in$ GL_LIST for the loop in KeyboardClassUnload (described in Section 2.1); it describes the type-state of the local variable entry.

Modifies clauses. In the absence of any annotation about the side-effect (or modifies set), a procedure havocs (scrambles) all the globals the fields and typestates of pointers in the heap. Modifies annotations are needed to provide precise updates to the relevant fields and type-states.

Conditional annotations. Sometimes the annotations in each of above categories need to be guarded by some conditions (e.g. return value, flags in some parameters). The procedure KbdCreateClassObject (encountered earlier in this section) creates and returns a MyDevObj pointer through the out-parameter *ClassDeviceObject, only when the return value is non-negative.

In the remainder of the paper, we formalize the notion of type-states (Section (3), and provide mechanisms to generate internal annotations guided by the module invariants (Section 4), and finally evaluate our technique on a set of real-world device drivers (Section [5).

\section{Type-State Assertions}

In this section, we formally define a type-state and type-state assertions that comprise our annotation language. A type-state is a set of pointers, and the type-state of a pointer is determined by membership or exclusion from the set. Type-states are a generalization of static types in a program and are useful 


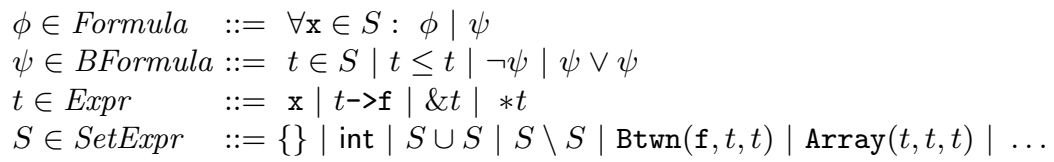

Fig. 3. Grammar for type-state invariants. Here $f$ refers to a program field. The toplevel invariant is of the form $\phi, S$ is a set expression, and int is the set of all integers. We require that $\mathrm{x}$ not appear in $S$ in the formula $\forall \mathrm{x} \in S: \phi$.

for two purposes: (a) for establishing non-aliasing between pointers belonging to disjoint type-states, and (b) to state a property about a set of pointers satisfying a type-state.

The language of type-states is extensible by the user and includes the following:

1. Static. The static types in programs correspond to immutable type-states.

2. Dynamic. Some type-states are mutable at runtime. For example, the typestate MyDevObj is mutated by calls to IoCreateDevice and IoDeleteDevice.

3. Membership in data structures. As we have seen, we often need to distinguish pointers based on their membership in a list (e.g., GLLIST), a tree or an array. We use set constructors to refer to all elements of a list between two pointers (Btwn) or all elements in the range between two pointers or integers [6].

Often, there are interesting sub-typing relationships among type-states. For example, the type-state MyDevObj is a subtype of PDEVICE_OBJECT.

Figure 3 shows the recursive structure of a type-state assertion. Formula represents a top-level assertion, that can either be a quantified type-state assertion, or a BFormula. A BFormula represents a Boolean combination over type-states and arithmetic relations over terms; Expr represents the various pointer terms that can be constructed from a variable x. The vocabulary of type-states SetExpr is extensible by the user. Here $\operatorname{Array}(a, s, n) \doteq\{a+s * i \mid 0 \leq i<n\}$ is the set constructor for all pointers in an array of size $n$ starting at $a$, where each entry has a size of $s$ bytes.

The quantified assertions represent type-state invariants which state a property for all pointers satisfying a given type-state; examples of such an assertion are invariants 11 and 5 in Figure 2 Quantifier-free assertions are used to describe type-states of variables or their fields; invariants 7 and 8 are examples of it. Type-state invariants can be nested: the following invariant establishes the parent-child relationship for all elements in a list:

$$
\forall \mathrm{x} \in \text { TypeA }: \forall \mathrm{y} \in \mathrm{Btwn}(\text { Flink, } \mathrm{x}->\text { first, NULL }): \mathrm{y}->\text { parent }=\mathrm{x}
$$

\section{Generating Internal Annotations}

Given the interface specification and the module invariants, the next step is to infer the internal annotations. There are two broad classes of annotations that 
need to be inferred for modular verification - assertions and modifies clauses. Assertions include loop invariants and preconditions and postconditions on internal procedures.

As we illustrated in Section 2, sound verification of real-world modules requires module invariants to contain quantifiers and Boolean connectives. Automatic inference of such annotations is challenging and existing procedures for constructing quantified invariants with Boolean structure is limited to relatively small programs 15/19 17/24]. In this section, we demonstrate the use of exception sets to synthesize a broad class of internal annotations containing quantifiers and Boolean structure, in a scalable fashion guided by the module invariants.

We use the Houdini 13 algorithm for inferring the internal annotations of a module decorated with module invariants. The algorithm is simple yet scalable: the algorithm takes as input a set of candidate annotations for the internal procedures and outputs the largest subset of the candidate annotations that is mutually consistent. The algorithm initially assumes all the candidate annotations and then greedily removes an annotation that does not hold during a modular verification step - the process terminates when the set of annotations is consistent or empty. Although this can be seen as solving a restricted case of the predicate abstraction problem (often called monomial predicate abstraction), the restricted nature of the problem makes it scalable. For example, when the complexity of deciding formulas in the assertion logic is Co-NP complete, the complexity of the predicate abstraction problem is PSPACE complete, whereas the complexity of monomial predicate abstraction is Co-NP complete [21.

The main challenge in using Houdini is to generate enough candidate annotations to capture a broad class of internal invariants containing quantifiers and Boolean connectives. In the next two sections, we illustrate the use of exception sets to generate candidate annotations for assertions (Section 4.1) and modifies clauses (Section 4.2) guided by the module invariants. We conclude the section with some description of conditional annotations that our approach does not capture currently (Section 4.3).

\subsection{Candidate Assertions}

We have found the following kinds of assertions suitable for populating the candidate pool for preconditions, postconditions, and loop invariants.

Type-states of pointers. The various type-states mentioned in the module invariants and the interface specification signify the relevant attributes of pointers that should be tracked. For every well-typed path expression in scope and for each relevant type-state, we introduce a candidate assertion stating that the path expression is in the type-state. The declared types of variables and fields are of great value in reducing the set of pointers to check for membership in a given type-state. These candidate facts are important for instantiating the universally-quantified type-state invariants in the module invariants.

Module invariant exceptions. Consider a module invariant $\forall \mathrm{x} \in S: \psi(\mathrm{x})$ which specifies some property $\psi(x)$ holds for all pointers $x$ satisfying $S$. Such 
an assertion could be broken at a few pointers, which we call module invariant exceptions. We guess that this can happen only for the sets of pointers $\Theta_{1}, \Theta_{2}, \ldots$, $\Theta_{k}$. We can generate the following candidate assertions:

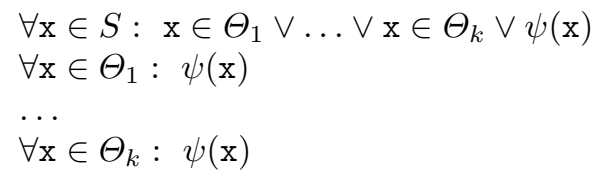

Assuming the different sets $\Theta_{1}, \ldots, \Theta_{k}$ are pairwise disjoint, the above candidate assertions allow us to capture the tighest exception to the module invariant in terms of the input sets. For instance, if the module invariant holds, then all the candidate assertions hold. On the other hand, if the exceptions to the module invariant cannot be captured in the input sets, then the first assertion would fail. When a set $\Theta_{i} \doteq\left\{\theta_{i}\right\}$ is a singleton, then the quantified fact $\forall \mathrm{x} \in \Theta_{i}: \psi(\mathrm{x})$ can be simplied to $\psi\left(\theta_{i}\right)$.

Example 1. Consider a simple example with the following module invariant in the "steady state" (recall the set constructor Array from Section 31):

$$
\forall \mathrm{x} \in \operatorname{Array}(\mathrm{a}, 4, \mathrm{n}): \mathrm{x}->\mathrm{d}=42
$$

For a loop that initializes the array, the loop invariant is a combination of a module invariant exception and a type-state assertion on the pointer iter, where iter is the iterator over the array.

$$
\begin{aligned}
& \forall \mathrm{x} \in \operatorname{Array}(\mathrm{a}, 4, \mathrm{n}): \mathrm{x} \in \operatorname{Array}(\mathrm{a}, 4, \mathrm{n}) \backslash \operatorname{Array}(\mathrm{a}, 4, \text { iter }) \vee x->\mathrm{d}=42 \\
& \text { iter } \in \operatorname{Array}(0,1, \mathrm{n}+1)
\end{aligned}
$$

The exception set in this example is $\operatorname{Array}(\mathrm{a}, 4, \mathrm{n}) \backslash \operatorname{Array}(\mathrm{a}, 4$, iter $)$.

\subsection{Candidate Modifies Clauses}

A candidate modifies annotation for a field $\mathrm{F}$ looks as follows:

$$
\begin{aligned}
& \text { modifies } \mathrm{F} \Theta_{1} \\
& \text { modifies } \mathrm{F} \Theta_{k}
\end{aligned}
$$

This is an annotation that specifies that $\mathrm{F}$ is possibly modified only at pointers in the set $\bigcup_{1 \leq i \leq k} \Theta_{i}$. These annotations relate the state of $\mathrm{F}$ at entry and exit from a procedure, or at entry and the beginning of an arbitrary iteration of a loop. Modifies annotations are crucial for tracking the side-effect of a procedure or a loop on the heap.

We model the memory as a collection of maps, one for each type and each field [8]. Notice that the modifies annotation simply says that the sets $\Theta_{i}$ are exceptions to the assertion stating that the map $\mathrm{F}$ is preserved at all pointers! Hence, we use an almost identical strategy as the module invariant exceptions. If the user provides the above modifies annotations as candidates, we generate the following candidate annotations: 


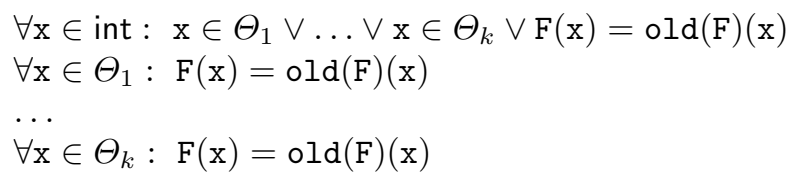

Here old $(F)$ refers to the value of the map $F$ at the pre-state (either at the entry of a procedure or a loop). For singleton $\Theta_{i}=\left\{\theta_{i}\right\}$, the quantified assertions can be simplified to $\mathrm{F}\left(\theta_{i}\right)=\mathrm{old}(\mathrm{F})\left(\theta_{i}\right)$.

The modifies clauses for mutable type-states (such as MyDevExtn) are dealt with similarly.

\subsection{Conditional Annotations}

In some cases, the above candidate assertions may need to be guarded by the type-states of a few pointers. For example, the type-state of a pointer might depend on the type-state of the return variable and/or some flag in the parameters. In other cases, different type-states of a pointer may be correlated (e.g., invariant 7 in Figure 2). We currently require the user to provide the conditional annotations.

\section{Implementation and Results}

We have implemented a prototype tool for the problem of intra-module inference. The input to the tool is a module (a single compilation unit) written in $\mathrm{C}$, along with the specifications of external procedures that are called from the module, and the interface specifications for the public procedures. The user then describes a set of module invariants (similar to Figure 2) for the module. Our tool generates a set of candidate annotations and infers the internal annotations over them using the Houdini algorithm.

Generation of the candidate annotations from the module invariants requires providing a set of pointers at procedure and loop boundaries. These pointers form parts of type-state assertions or exception sets for module invariants and modifies clauses. Pointers can range over the variables (procedure parameters, globals) in scope and field dereferences over them. However, manually specifying the pointers for each procedure and loop can be cumbersome. To relieve this burden, we provide patterns that match against the variables in scope at procedure and loop boundaries to generate actual pointer expressions. For example, to generate pointers of type PDEVICE_EXTENSION, we provide a pattern to match against variables of type PDEVICE_EXTENSION, or DeviceExtension fields of PDEVICE_OBJECT variables.

We use HAVOC to translate [8] an annotated $\mathrm{C}$ program into an intermediate language BoogiePL 12 and use the Boogie [4] verification condition generator to translate a BoogiePL program into an logical formula. We use an efficient implementation of the Houdini algorithm [13] using the Satisfiability Modulo Theory (SMT) solver Z3 [1]. 


\subsection{Benchmarks}

We have evaluated our prototype on 4 sample device driver modules in the Windows operating system. These drivers are distributed with the Windows Driver Development Kit (DDK) 1]. Among these drivers, kbdclass is a class driver for keyboard devices installed in a system, mouclass is a class driver for all mouse devices installed in a system, flpydisk is a class floppy driver, and mouser is a serial mouse driver. The size and complexity of the drivers are mentioned in Figure 4.

\begin{tabular}{|c|c|c|c|c|}
\hline Example & LOC & \# Pr & \# Pub & \# Loops \\
\hline kbdclass & 7242 & 51 & 28 & 20 \\
\hline mouclass & 6857 & 50 & 27 & 18 \\
\hline flpydisk & 6771 & 35 & 11 & 24 \\
\hline mouser & 7415 & 67 & 27 & 12 \\
\hline
\end{tabular}

\begin{tabular}{|c|c|c|c|c|c|c|c|c|}
\hline Example & \multicolumn{2}{|c|}{$\begin{array}{c}\text { Module Inv } \\
\text { Type }\end{array}$} & Glob & & \multicolumn{2}{|c|}{ Manual } & \multicolumn{2}{c|}{ Time } \\
& Pr & Cond & Oth & Inf & Chk \\
\hline kbdclass-df & 5 & 3 & 1476 & 3 & 1 & 2 & 480 & 190 \\
kbdclass-all & 6 & 3 & 1591 & 3 & 4 & 2 & 818 & 228 \\
\hline mouclass-df & 5 & 3 & 1391 & 3 & 1 & 2 & 491 & 185 \\
mouclass-all & 6 & 3 & 1502 & 3 & 4 & 2 & 892 & 273 \\
\hline flpydisk-df & 4 & 0 & 1355 & 0 & 0 & 0 & 632 & 129 \\
flpydisk-all & 5 & 0 & 1431 & 0 & 0 & 0 & 827 & 167 \\
\hline mouser-df & 4 & 0 & 1608 & 0 & 0 & 0 & 571 & 126 \\
mouser-all & 5 & 0 & 1774 & 2 & 0 & 2 & 224 & 124 \\
\hline \hline
\end{tabular}

Fig. 4. Benchmarks and results: "df" extensions check double-free and "all" checks both the double-free and lock-usage property. "Module Inv" is the number of module invariants, and comprises of type-state invariants ("Type") type-state assertions on globals ("Glob"). "Infrd" and "Manual" represent sizes of inferred, and manual annotations. "Pr" is the number of procedures manually annotated. The manual annotations are broken up into conditional ("Cond") or others ("Oth"). "Time" is the runtime in seconds - "Inf" is the time for inference, and "Chk" is the time to check the annotated program.

For each driver, we check two properties:

1. The double-free property, as illustrated in Section 2.

2. The lock-usage property, as described below.

The lock-usage property states that all KSPINLOCK locks alternate between acquired and released states by calls to KeAcquireSpinLock and KeReleaseSpinLock respectively, after the lock has been initialized into the released state by a call to KeInitializeSpinLock. We use a mutable type-state Released to capture the state of a lock; this type-state is modified by these Ke*SpinLock procedures.

Since these locks appear as fields of device extensions, we also augmented the module invariants with the following invariant:

$$
\forall \mathrm{x} \in \text { MyDevExtn : } \bigwedge_{i} \text { Released }\left(\& \mathrm{x}^{->} \text {lock }_{i}\right. \text { ) }
$$

where lock $_{i}$ is the name of the $i^{\text {th }}$ field of type KSPINLOCK. This invariant signifies that at entry and exit from a module, the locks in the module are released.

\section{$5.2 \quad$ Results}

Figure 4 summarizes the results of our experiments. The experiments were performed on a $2.4 \mathrm{GHz}$ machine running Windows with $2 \mathrm{~GB}$ of memory. For each 
driver, we first check the double free property (-df extension) and then check both the properties (-all extension). For checking the -all properties, the additional type-state invariant ("Type") in the module invariant column refers to the invariant $\mathrm{L}$ described for the locks. Since flpydisk and mouser do not have any global variables relevant to the properties, their module invariants do not require any type-state assertions on the globals.

The results show that our technique is able to infer most of the internal annotations - the number of manual annotations is small. Besides, the number of inferred annotation is much larger than the set of module invariants, thereby justifying the intra-module inference problem. Finally, our inference technique is scalable; the time taken to generate the inferred annotations ("Inf") is of the same order as the time taken to check the final set of annotations ("Chk"). A distributed implementation of the algorithm would further reduce the time.

We have already seen that the burden of writing the module invariants is low, and these annotations are quite succinct. In fact, most of the module invariants for MyDevObj and MyDevExtn are reusable across all drivers of a given class, modulo renaming of fields. This further amortizes the annotation effort across a class of drivers, and also provides module invariants that drivers of a given class should document and export.

Some internal annotations still had to be provided manually. For kbdclass, most of the manual annotations were on a procedure KbdCreateClassObject which is a wrapper around IoCreateDevice with partial initialization of some of the fields. These annotations were guarded postconditions, where the guard is a predicate on the return value signifying successful device creation. More such annotations were needed for checking the lock-usage property, as only a subset of the locks were initialized in the procedure violating the module invariant $\mathrm{L}$ temporarily on some locks. The other manual annotations required for this module included the loop invariant entry $\in$ GL_LIST for the KeyboardClassUnload 1 For mouser, the additional annotations for checking both the properties come from the need to specify preconditions on the objects of asynchronous procedure calls that are never called directly — these procedures get enqueued along with their input argument (a DEVICE_EXTENSION object), and can fire later. For these procedures, we had to specify that the argument satisfied the type-state MyDevExtn.

For mouser, Figure 4 shows a surprising result: the runtime of the inference component of mouser-all is substantially smaller than mouser-df, even though the latter checks only one property. Recall that the verification of mouser-all required extra manully-provided preconditions on asynchronous procedure calls. Even though the verification of mouser-df did not require these preconditions, their absence caused many candidate annotations to be refuted in Houdini and thus the algorithm took longer to converge.

The verification of kbdclass and mouclass relies on an assumption that has not been mechanically verified. These drivers have linked lists of two different

\footnotetext{
${ }^{1}$ This loop invariant matches our template for candidate assertions, but our tool currently does not support instrumenting local variables. Therefore, the user has to provide loop invariants involving local variables.
} 
types, DEVICE_EXTENSION and IRP, that use the same underlying Flink field. We assume that linked lists of these two types are disjoint. We have verified this assumption manually, and are currently working on verifying it mechanically.

\section{Related Work}

In this work, we have shown how to extend the precise reasoning performed by modular checkers to large modules in systems code with small annotation overhead. We have shown that verification of simple type-state properties may need invariants about type-states and data structures that can be best specified by users at the level of a module. In this section, we briefly compare with related work on checking type state properties on large modules.

Automated software verification tools for simple type-state properties (e.g. lock-usage, resource leaks) are largely based on predicate abstraction [16] (e.g. SLAM [3], BLAST [18]), data-flow analysis (e.g. ESP [10], Saturn [2]) and more recently on interpolants 23 . Most of these approaches lose precision when the analysis requires complex type-state invariants in the presence of open modules or unbounded heap, as shown in Figure 2, resulting in false alarms. Existing automated tools deal with this problem in two ways. First, post processing of the set of warnings is done to heuristically report a subset of warnings whereby real bugs may be hidden. Second, a harness is created that nondeterministically calls a public procedure after initializing the heap with a small number of objects, thus avoiding the need to specify quantified invariants on an unbounded set of heap objects. Both of these approaches reduce false alarms by sacrificing soundness.

Tools based on abstract interpretation [9] have been specialized to perform shape analysis 25] for systems code. These tools have specialized abstractions to deal with recursive data structures. Recent work using separation logic has been shown to scale to realistic device drivers [26. However, such tools cannot be used for checking generic type-state properties without building specialized abstract domains. Unlike our method, these tools do not allow the specification and verification of the module invariants.

Verification of object-oriented programs makes heavy use of class or module invariants 4]. However, the focus on annotation inference and automation is secondary - these techniques are primarily focused on scalability of inter module analysis, where each module has manageable complexity. However, for OS modules with several hundred and possibly thousands of internal procedures, the problem of intra-module inference is crucial for utilizing module invariants. The work on HOB 22 is closest in spirit to our work, where a combination of user-specified type-state annotations and inference is used to verify non-trivial programs; however unlike our approach the inference does not leverage the module invariants.

\section{Acknowledgments}

We wish to thank Aditya Nori, Julien Vanegue, and the anonymous reviewers for their detailed comments. 


\section{References}

1. Windows Driver Kit, http://www.microsoft.com/whdc/devtools/wdk/default.mspx

2. Aiken, A., Bugrara, S., Dillig, I., Dillig, T., Hackett, B., Hawkins, P.: An overview of the Saturn project. In: Program Analysis for Software Tools and Engineering (PASTE 2007), pp. 43-48. ACM, New York (2007)

3. Ball, T., Majumdar, R., Millstein, T., Rajamani, S.K.: Automatic predicate abstraction of $\mathrm{C}$ programs. In: Programming Language Design and Implementation (PLDI 2001), pp. 203-213 (2001)

4. Barnett, M., Leino, K.R.M.: Weakest-precondition of unstructured programs. In: Program Analysis For Software Tools and Engineering (PASTE 2005), pp. 82-87. ACM, New York (2005)

5. Barnett, M., Leino, K.R.M., Schulte, W.: The Spec\# programming system: An overview. In: Barthe, G., Burdy, L., Huisman, M., Lanet, J.-L., Muntean, T. (eds.) CASSIS 2004. LNCS, vol. 3362, pp. 49-69. Springer, Heidelberg (2005)

6. Chatterjee, S., Lahiri, S.K., Qadeer, S., Rakamarić, Z.: A reachability predicate for analyzing low-level software. In: Grumberg, O., Huth, M. (eds.) TACAS 2007. LNCS, vol. 4424, pp. 19-33. Springer, Heidelberg (2007)

7. Clarke, E.M., Grumberg, O., Peled, D.A.: Model Checking. MIT Press, Cambridge (2000)

8. Condit, J., Hackett, B., Lahiri, S.K., Qadeer, S.: Unifying type checking and property checking for low-level code. In: Principles of Programming Languages (POPL 2009), pp. 302-314 (2009)

9. Cousot, P., Cousot, R.: Abstract interpretation: A unified lattice model for the static analysis of programs by construction or approximation of fixpoints. In: Symposium on Principles of Programming Languages (POPL 1977), pp. 238-252. ACM Press, New York (1977)

10. Das, M., Lerner, S., Seigle, M.: ESP: Path-sensitive program verification in polynomial time. In: Programming Language Design and Implementation (PLDI 2002), pp. 57-68 (2002)

11. de Moura, L., Bjorner, N.: Efficient incremental e-matching for SMT solvers. In: Pfenning, F. (ed.) CADE 2007. LNCS, vol. 4603, pp. 183-198. Springer, Heidelberg (2007)

12. DeLine, R., Leino, K.R.M.: BoogiePL: A typed procedural language for checking object-oriented programs. Technical Report MSR-TR-2005-70, Microsoft Research (2005)

13. Flanagan, C., Leino, K.R.M.: Houdini, an annotation assistant for ESC/Java. In: Oliveira, J.N., Zave, P. (eds.) FME 2001. LNCS, vol. 2021, pp. 500-517. Springer, Heidelberg (2001)

14. Flanagan, C., Leino, K.R.M., Lillibridge, M., Nelson, G., Saxe, J.B., Stata, R.: Extended static checking for Java. In: Programming Language Design and Implementation (PLDI 2002), pp. 234-245 (2002)

15. Flanagan, C., Qadeer, S.: Predicate abstraction for software verification. In: Principles of Programming Languages (POPL 2002), pp. 191-202. ACM Press, New York (2002)

16. Graf, S., Saïdi, H.: Construction of abstract state graphs with PVS. In: Grumberg, O. (ed.) CAV 1997. LNCS, vol. 1254, pp. 72-83. Springer, Heidelberg (1997)

17. Gulwani, S., McCloskey, B., Tiwari, A.: Lifting abstract interpreters to quantified logical domains. In: Principles of Programming Languages (POPL 2008), pp. 235246 (2008) 
18. Henzinger, T.A., Jhala, R., Majumdar, R., Sutre, G.: Lazy abstraction. In: Principles of Programming Languages (POPL 2002), pp. 58-70 (2002)

19. Lahiri, S.K., Bryant, R.E.: Constructing quantified invariants via predicate abstraction. In: Steffen, B., Levi, G. (eds.) VMCAI 2004. LNCS, vol. 2937, pp. 267-281. Springer, Heidelberg (2004)

20. Lahiri, S.K., Qadeer, S.: Back to the future: revisiting precise program verification using SMT solvers. In: Principles of Programming Languages (POPL 2008), pp. 171-182 (2008)

21. Lahiri, S.K., Qadeer, S.: Complexity and algorithms for monomial and clausal predicate abstraction. Technical Report MSR-TR-2009-2012, Microsoft Research (2009)

22. Lam, P., Kuncak, V., Rinard, M.C.: Generalized typestate checking for data structure consistency. In: Cousot, R. (ed.) VMCAI 2005. LNCS, vol. 3385, pp. 430-447. Springer, Heidelberg (2005)

23. McMillan, K.L.: Lazy abstraction with interpolants. In: Ball, T., Jones, R.B. (eds.) CAV 2006. LNCS, vol. 4144, pp. 123-136. Springer, Heidelberg (2006)

24. McMillan, K.L.: Quantified invariant generation using an interpolating saturation prover. In: Ramakrishnan, C.R., Rehof, J. (eds.) TACAS 2008. LNCS, vol. 4963, pp. 413-427. Springer, Heidelberg (2008)

25. Sagiv, S., Reps, T.W., Wilhelm, R.: Solving shape-analysis problems in languages with destructive updating. ACM Transactions on Programming Languages and Systems (TOPLAS 1998) 20(1), 1-50 (1998)

26. Yang, H., Lee, O., Berdine, J., Calcagno, C., Cook, B., Distefano, D., O'Hearn, P.W.: Scalable shape analysis for systems code. In: Gupta, A., Malik, S. (eds.) CAV 2008. LNCS, vol. 5123, pp. 385-398. Springer, Heidelberg (2008) 